\title{
Evaluación de la capacidad de mineral aluminosilicato de la localidad de Cacha para la remoción de color en efluentes de la industria de lavado de blue jeans
}

\author{
Evaluation of the aluminosilicate mineral capacity of the town of Cacha for the \\ removal of color in effluents from the blue jeans washing industry
}

Andrés Lara Hernández. ${ }^{1}$, Hugo Calderón. ${ }^{2}$, Ma. Eugenia Ramos Flores ${ }^{3}$. \& Marcelo Ramos Flores. $^{4}$

\begin{abstract}
DOI: https://doi.org/10.33262/cienciadigital.v3i3.654

Effluent contamination affects freshwater bodies due to its low capacity for selfpurification and limitation for the diffusion of light for photosynthetic processes; This problem is notorious in populations where the textile industry uses traditional methods of dyeing and washing, such as Pelileo and Guano. The use of clayey aluminosilicate minerals has become relevant in recent years as a low-cost alternative and lower environmental footprint alternative for the development of adsorbents for the removal of dyes; so his study is of interest in developing countries. The present investigation presents the achievements of the application of four adsorbents developed from aluminosilicate ore from Cacha population, province of Chimborazo, one in its natural state and the rest obtained by acid, basic and thermal modification, on effluent from a laundry jeans from Pelileo town. The results show that, in all cases, the use of these adsorbent achieves compliance with demands of TULSMA; however, the highest percentages of removal occurred in the thermal and basic treatments. The results show the viability of the use of clay-type aluminosilicate minerals as a basis for the development of technologies for the removal of polluting waste.
\end{abstract}

Key Words: Aluminosilicates, Dye Removal, Textile Industry, Indigo Blue, Adsorbents

\footnotetext{
${ }^{1}$ Escuela Superior Politécnica de Chimborazo, Chimborazo Ecuador, kurtpanda@live.com

${ }^{2}$ Escuela Superior Politécnica de Chimborazo, Chimborazo Ecuador, hcalderon@espoch.edu.ec

${ }^{3}$ Universidad Estatal Amazónica. Pastaza, Ecuador, mramos@uea.edu.ec

${ }^{4}$ Escuela Superior Politécnica de Chimborazo, Chimborazo Ecuador,jmramos@espoch.edu.ec
} 


\section{Resumen}

La contaminación de efluentes afecta los cuerpos de agua dulce por su baja capacidad de autodepuración y limitación para el ingreso de luz para procesos fotosintéticos; este problema es notorio en poblaciones donde la industria textil usa métodos tradicionales de tinturado y lavado, como Pelileo y Guano. El uso de minerales aluminosilicatos de tipo arcilloso ha tomado relevancia en los últimos años como alternativa de bajo costo y menor huella ambiental para el desarrollo de adsorbentes para remoción de colorantes; por lo que su estudio es de interés en países en vías de desarrollo. La presente investigación presenta los resultados de la aplicación de cuatro adsorbentes desarrollados a partir de mineral aluminosilicato de la localidad de Cacha, provincia de Chimborazo, uno en estado natural y los restantes obtenidos por modificación ácida, básica y térmica, sobre efluente de una lavandería de jeans del cantón Pelileo. Los resultados mostraron que, en todos los casos, el uso de estos adsorbente logra el cumplimiento de lo indicado en el TULSMA; no obstante, los mayores porcentajes de remoción se dieron en los tratamientos térmico y básico. Los resultados evidencian la viabilidad del uso de minerales aluminosilicatos de tipo arcilloso como base para el desarrollo de tecnologías para la remoción de residuos contaminantes.

Palabras Clave: Aluminosilicatos, Remoción de Color, Industria Textil, Azul Índigo, Adsorbentes.

\section{Introducción}

Dentro de la industria textil es usual el uso de procesos denominados "húmedos", que abarcan operaciones de desengomado, focalizado, acabados, enjuague y tinturado (Moreno, 2007). No obstante, su consumo de agua y generación de efluentes son elevados, llegando a afectar la vida acuática (Cortazar Martínez, Coronel Olivares, Escalante, \& González Ramírez, 2014). En el caso particular del lavado y tinturado, se estima que el consumo de agua llega a diez litros por cada kilogramo de ropa y, respecto al colorante, llega a 15 kilogramos de colorante diluido por cada kilogramo de ropa (Llive Carrillo, 2012).

Los colorantes de mayor uso son los del tipo sintético, particularmente el azul índigo (Garzón, 2009). Las aguas residuales con colorantes son consideradas como basura industrial y usualmente se las descarga a los recursos hídricos que rodean a las empresas sin un tratamiento previo (Yagub, Sen, Afroze, \& Ang, 2014). El colorante descargado a cuerpos de agua dulce es un problema ambiental por su baja capacidad de autodepuración (Sanz, 2015), y prácticamente nula biodegradabilidad (Ngulube, Gumbo, Masindi, \& Maity, 2017a). Por otro lado, la presencia de color limita la transmisión de luz solar, afectando a la actividad fotosintética que tenga lugar en lagos y ríos (Re, 2010). 
En los últimos años los métodos de tratamientos de efluentes están buscando alternativas de bajo costo y menor huella ambiental, tal como los minerales aluminosilicatos (Ngulube et al., 2017a). Estos minerales son complejos de filosilicatos hidratados con aluminio, que presentan plasticidad al humedecerse y que alcanzan dureza al ser sometidos a temperaturas de calcinación o secado (De Pablo, 1964); su estructura, a nivel químico incluye presencia de capas tetraédricas $(\mathrm{SiO} 4)^{4-}$ y octaédricas $\left(\mathrm{AlO}_{6} / \mathrm{MgO}_{6}\right)($ Martínez, 2017), que forman estructuras regulares que les otorgan elevada área superficial y alta capacidad de adsorción e intercambio de iones, características ideales para potenciales materiales para tratamientos económicos y sostenibles (Ngulube, Gumbo, Masindi, \& Maity, 2017b).

Adicional a sus características estructurales, elevada área superficial y capacidad de intercambio iónico. Los minerales aluminosilicatos, en particular las arcillas, son susceptibles de modificación por tratamientos físicos o químicos, que aumenta su área superficial, porosidad del material y grupos funcionales en superficie, permitiendo la aparición de más sitios activos para la unión de los iones del colorante con la superficie del adsorbente (Kausar et al., 2018a).

\section{Importancia del Problema}

En Ecuador las aguas de descarga deben cumplir con los parámetros registrados en TULSMA, libro VI, anexo I, tabla 9. En lo referente a color, este debe ser inapreciable a dilución 1:2 (Ministerio del Ambiente, 2015).

Investigaciones realizadas en la sierra centro de Ecuador, particularmente en los cantones de Pelileo y Guano, han determinado que su pujante industria textil, que utiliza métodos tradicionales de tintura y lavado, genera aguas residuales con valores altos de DBO, DQO, pH y color, con afectación a los ríos, en especial ocurriendo la alteración por colorantes. (Redacción Sierra Centro, 2010).

El uso de colorantes textiles es amplio, llegando a miles de diferentes formulaciones, de las cuales aproximadamente un $70 \%$ son azocolorantes. Además, la eficacia de las operaciones de tinturado y lavado es menor a la otras etapas de producción, determinándose que un $10 \%$ de colorante se pierde en los procesos de tinturado y hasta un 20\% llega a las aguas residuales (Arora, 2014).

Para la remoción de colorantes, la adsorción es considerada como la tecnología más viable y capaz de retener todos los colorantes (Kausar et al., 2018a); pero con la desventaja de los costos de los adsorbentes comerciales y el trámite para su disposición posterior. En este contexto, las arcillas y minerales aluminosilicatos se han convertido en materiales de interés, al ser considerados por sus características ya mencionadas como adsorbentes económicos, disponibles, eficientes y oportunos para países en vías de desarrollo (Ngulube et al., 2017a).

La presencia de minerales de tipo aluminosilicato y arcillosos en Ecuador es amplia. Según la Agencia de Regulación y Control Minero, ARCOM, en el año 2015 se produjo un estimado de 
479662 toneladas (Larenas Herdoíza, Fierro-Renoy, \& Fierro-Renoy, 2017), ocupando el segundo lugar, luego de las calizas (Ministerio de Minería de Ecuador, 2016). En la zona 3, que comprende las provincias de Cotopaxi, Chimborazo, Tungurahua y Pastaza, según reportes del Instituto Nacional de Investigación Geológico Minero Metalúrgico, INIGEMM, se tienen identificados 34 afloramientos o frentes de explotación de aluminosilicatos tipo arcilla (Ministerio de Minería de Ecuador, 2016) (Uribe, 2015).

De lo anterior, resulta evidente el interés y utilidad de una investigación de la remoción de colorantes basada en el uso de aluminosilicatos. Enfocándose la presente investigación al caso al tratamiento de efluentes de lavandería de jeans, cuyas aguas azuladas contienen compuestos de azul índigo, aplicando como adsorbente mineral aluminosilicato proveniente del sector de Cacha, provincia de Chimborazo, aplicado tanto en estado natural como modificado por tratamiento ácido, básico y térmico.

\section{Metodología}

\section{A. Muestreo de efluente textil}

El muestreo de efluente siguió la norma NTE-INEN 2169:98, utilizando botellas plásticas de 2,0 L de capacidad por recolección directa del flujo de descarga (INEN, 2013). Se realizó un total de 6 tomas de muestra durante los tiempos de liberación de efluente de una lavandería de jean ubicada en el cantón Pelileo, cuyo nombre, por petición expresa de los propietarios, se mantiene en reserva. La tabla de muestreo se indica a continuación.

Tabla 1: Tomas de muestra de efluente

\begin{tabular}{|c|c|c|}
\hline $\begin{array}{l}\text { Toma } \\
\text { muestra }\end{array}$ & $\begin{array}{l}\text { Volumen } \\
\text { (L) }\end{array}$ & $\begin{array}{l}\text { Tiempo de llenado } \\
\text { (s) }\end{array}$ \\
\hline 1 & 2,0 & $7,30 \mathrm{~s}$ \\
\hline 2 & 2,0 & $6,75 \mathrm{~s}$ \\
\hline 3 & 2,0 & $7,15 \mathrm{~s}$ \\
\hline 4 & 2,0 & $6,45 \mathrm{~s}$ \\
\hline 5 & 2,0 & $6,84 s$ \\
\hline 6 & 2,0 & $7,62 \mathrm{~s}$ \\
\hline
\end{tabular}

Realizado por: Autores, 2018

\section{B. Determinación de color}

La medición del color se realizó basándose en los estándar APHA-AWWA-WPCF para medición de color, utilizando un fotómetro Hach 25, con calibración inicial utilizando agua 
destilada. Expresando los resultados en unidades platino-cobalto "PtCo" (APHA, AWWA, \& WPCF, 1989).

La medición de color real del efluente de lavandería se realizó sobre muestras de 10,0 mL de una dilución 1:3 de la muestra. La medición de color posterior a la aplicación del tratamiento con adsorbente se realizó sobre muestras de 10,0 mL tomadas del líquido sobrenadante diluido en proporción 1:3.

\section{Muestreo y reducción primaria del mineral}

Las muestras de mineral fueron tomadas en la localidad de Cacha, operando de acuerdo a lo indicado en la norma NTE-INEN 695:2010, que incluye inspección de la superficie del afloramiento, registro de variaciones texturales, remoción de corteza del afloramiento, realización de tres perforaciones en tres lugares diferentes del afloramiento tomando un mínimo de $2 \mathrm{~kg}$ de muestra en cada sitio excavado $(695,2010)$. La muestra bruta fue almacenada en bolsas plásticas y luego, en laboratorio, triturada y tamizada a luz de malla de $150 \mu \mathrm{m}$ puesto que un tamaño reducido de partícula favorece la capacidad de adsorción (Saif Ur Rehman et al., 2013).

\section{Modificaciones sobre el mineral}

\section{Modificación ácida}

Se trabajó con una proporción de 10 g de mineral colocado en $100 \mathrm{~mL}$ de $\mathrm{HCl}$ 0,5 M. La mezcla se agitó durante $20 \mathrm{~min}$ a una temperatura de $25^{\circ} \mathrm{C}$. Luego, se dejó sedimentar, midió el pH del sobrenadante y lavó el sedimento, hasta obtener un sobrenadante cercano a la neutralidad. Posteriormente, se filtró el mineral al vacío, secó por $12 \mathrm{~h}$ a $60{ }^{\circ} \mathrm{C}$, trituró y tamizó a luz de malla de $150 \mu \mathrm{m}$. Las condiciones de modificación ácida fueron definidas por triangulación de múltiples publicaciones, entre ellas las de Ajemba, Dikmen, Silva, Ugurlu, y Zaghouane (Ajemba, Igbokwe, \& Onukwuli, 2012; Dikmen, Yilmaz, Yorukogullari, \& Korkmaz, 2012; Silva, Linares, Vicuña, \& Garrido, 2016; Uğurlu, 2009; Xavier et al., 2014; Zaghouane-Boudiaf, Boutahala, Sahnoun, Tiar, \& Gomri, 2014)

\section{Modificación básica}

Se utilizó $\mathrm{Na}(\mathrm{OH}) 3 \mathrm{M}$, en una proporción de $100 \mathrm{~mL}$ de hidróxido sobre 10,0 g de mineral, con agitación durante 20 minutos a $25^{\circ} \mathrm{C}$. De manera similar al tratamiento ácido, de manera sucesiva se dejó sedimentar, midió el pH del sobrenadante, descartó el sobrenadante y lavó el sedimento, hasta que el sobrenadante presentó un $\mathrm{pH}$ cercano a la neutralidad. Posteriormente se filtró la muestra al vacío, secó a $60{ }^{\circ} \mathrm{C}$ por $12 \mathrm{~h}$. para, finalmente, triturar y tamizar a luz de malla de $150 \mu \mathrm{m}$. Las condiciones de tratamiento básico se definieron por comparación de las investigaciones de Akgul, Ghosh, Ngulube, Stawinski y Wang (Akgül \& Karabakan, 2011; 
Crini, 2015; Ghosh \& Bhattacharyya, 2002; Ngulube et al., 2017a; Stawiński et al., 2017; Wang, Wang, Kang, \& Wang, 2015).

\section{Modificación térmica}

Se realizó en estufa a temperatura constante de $100^{\circ} \mathrm{C}$, secando una cantidad de $10 \mathrm{~g}$ de mineral durante 90 minutos. Esto, en base a información de investigaciones de Heller, Iyim, Khan y Toor (Heller-Kallai, 2013a; Iyim \& Güçlü, 2009; Khan, Dahiya, \& Ali, 2012; Toor, Jin, Dai, \& Vimonses, 2015).

\section{Mineral sin modificar, en estado natural}

Se utilizó mineral aluminosilicato, sin ningún tratamiento físico o químico que pueda alterar su composición o estructura; únicamente, se secó a temperatura constante de $60^{\circ} \mathrm{C}$, molió y tamizó a luz de malla de $150150 \mu \mathrm{m}$.

\section{E. Pruebas de Tratabilidad}

Los cuatro tipos de adsorbentes desarrollados (mineral natural, modificado por ácido, modificado por base y modificado térmicamente) se aplicaron para adsorción del color de muestras de efluente de lavandería de jeans, siguiendo el siguiente procedimiento, que fue concertado en base a las experiencias publicadas por Bingol, Hor, Toor y Turabik: a temperatura ambiente se colocaron $0,1 \mathrm{~g}$ de adsorbente sobre $100 \mathrm{~mL}$ de efluente previamente filtrado, agitando de manera constante durante 2 h., dejando reposar por $1 \mathrm{~h}$. y tomando del líquido sobrenadante, el que se diluyó en proporción 1:3 para realizar la lectura de color (Bingol, Tekin, \& Alkan, 2010; Hor et al., 2016; Toor \& Jin, 2012; Turabik, 2008)

\section{Resultados}

La remoción lograda por aplicación de cada adsorbente basado en mineral aluminosilicato y su estado de cumplimiento de la norma (TULSMA) antes y después del tratamiento, se muestran en la tabla 2 .

Tabla 2: Remoción promedio de color de efluente de lavandería de jeas, por aplicación de aluminosilicatos natural y modificados

\begin{tabular}{lllllll}
\hline & Cumplimiento & & & & \multicolumn{2}{c}{ Cumplimiento } \\
Modificación & $\begin{array}{l}\text { de la norma, antes } \\
\text { del tratamiento }\end{array}$ & $\mathbf{P C U}_{\mathbf{i}}$ & $\mathbf{P C U}_{\mathbf{f}}$ & $\begin{array}{l}\text { \% } \\
\text { Remoción }\end{array}$ & $\begin{array}{l}\text { de la norma, } \\
\text { después } \\
\text { tratamiento }\end{array}$ \\
\hline Ácida & No & & & dé \\
Térmica & No & 1065,0 & 939,5 & $11,8 \%$ & Sí \\
\hline
\end{tabular}




\begin{tabular}{llllll}
\hline $\begin{array}{l}\text { Básica } \\
\text { Ninguna } \\
\text { (estado }\end{array}$ & No & 1069,0 & 876,0 & $18,1 \%$ & Sí \\
natural) & No & 1067,5 & 960,5 & $10,0 \%$ & Sí \\
\hline
\end{tabular}

Realizado por: Autores, 2018

La comparación entre los resultados de los diferentes adsorbente se realizó aplicando análisis ANOVA de 1 factor, obteniéndose los siguientes resultados:

Tabla 3: ANOVA para comparación de los diferentes adsorbente basados en aluminosilicato

\begin{tabular}{|c|c|c|c|c|}
\hline Modificación & $\mathbf{N}$ & Medias & $\begin{array}{l}\text { Desviación } \\
\text { Estándar }\end{array}$ & $95 \% ; \mathrm{CI}$ \\
\hline Ácida & 6 & 11,8 & 1,060 & $\begin{array}{l}(10,010 ; \\
13,558)\end{array}$ \\
\hline Térmica & 6 & 19,7 & 2,341 & $\begin{array}{l}(17,913 ; \\
21,461)\end{array}$ \\
\hline Básica & 6 & 18,1 & 2,265 & $\begin{array}{l}(16,283 ; \\
19,830)\end{array}$ \\
\hline Natural & 6 & 10,0 & 2,369 & $\begin{array}{l}(8,244 ; \\
11,792)\end{array}$ \\
\hline
\end{tabular}

Realizado por: Autores, 2018

Gráfico 1. Diagrama de cajas del porcentaje de remoción obtenido con diferentes modificaciones al mineral aluminosilicato de Cacha

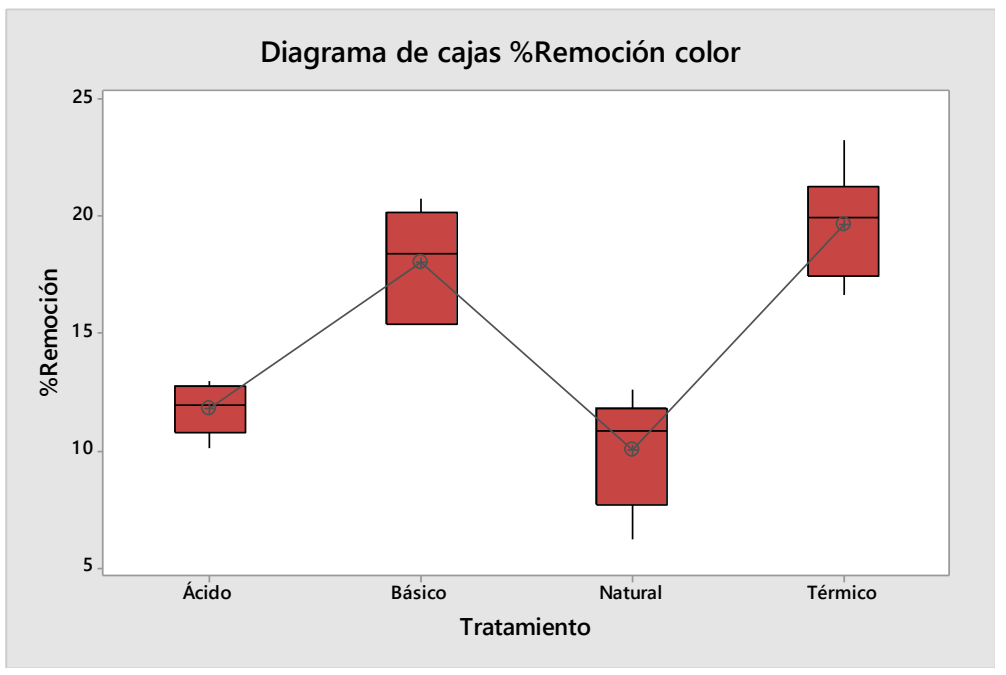

Fuente: Autores, Ecuador, 2017 


\section{Discusión}

Los resultados indican que la aplicación de adsorbentes, en todos los casos, logra una reducción del color que permite el cumplimiento de la norma. No obstante, el adsorbente que presenta mayor remoción de color, en las condiciones de experimentación, fue el mineral tratado térmicamente, con un 19,7\%. Este resultado puede deberse a que el tratamiento térmico, además de deshidratar la muestra, generarán espacios intercalados por la eliminación de agua incluida y ocluida. El efecto térmico sobre los aluminosilicatos ocurre en dos etapas: crecimiento del núcleo del material y proceso de difusión con elevadas energías de activación. (Heller-Kallai, 2013b; Toor et al., 2015)

En el caso del mineral modificado por tratamiento básico, sus valores de remoción son próximos a los del mineral modificado térmicamente, con remoción promedio del 18,1\%. Esto puede explicarse porque el tratamiento con bases permite la generación de espacios libres en el mineral aluminosilicato y la activación de grupos hidroxilo en superficie que generan atracción a los cationes del colorante. (Hor et al., 2016)

Los adsorbentes basado en mineral natural y modificado por ácido presentan las menores remociones. Este comportamiento puede deberse al hecho que, en estado natural, el mineral no posee suficientes sitios activos para la deposición del color a nivel superficial (Kausar et al., 2018b); y en el caso del adsorbente por modificación ácida, hay escaso intercambio entre hidrogeniones del colorante con cationes del mineral, lo cual limita la adsorción (Komadel \& Madejová, 2013).

\section{Conclusiones}

- El tratamiento térmico sobre mineral aluminosilicato de la localidad de Cacha permite la obtención de un adsorbente viable para la remoción de color en efluentes de lavanderías de jeans, con capacidad de remoción de un 19,7\%.

- Otro adsorbente viable, con un rendimiento ligeramente menor, se tiene en el adsorbente basado en aluminosilicato modificado por hidróxido.

- El mineral aluminosilicato utilizado sin tratar o con tratamiento ácido presenta menor rendimiento para la remoción de color de efluentes de lavanderías de jeans, con respecto a sus contrapartes obtenidas por tratamiento térmico y ácido.

- Los cuatro adsorbentes fueron efectivos en la remoción de color del efluente estudiado, con una diferencia entre los de mayor y menor rendimiento de un 9,7\%. Por lo que el estudio y desarrollo de tecnologías complementarias para el tratamiento de aguas basado en uso de minerales arcillosos se vislumbra como un campo viable para la utilización no-convencional y valorización de este tipo de minerales. 


\section{Referencias Bibliográficas}

Akgül, M., \& Karabakan, A. (2011). Promoted dye adsorption performance over desilicated natural zeolite. Microporous and Mesoporous Materials, 145(1-3), 157-164. https://doi.org/10.1016/j.micromeso.2011.05.012

APHA, AWWA, \& WPCF. (1989). Métodos normalizados para el análisis de aguas potables y residuales. (S. A. Diaz de Santos, Ed.).

Arora, S. (2014). Textile Dyes: It's Impact on Environment and its Treatment. Journal of Bioremediation \& Biodegradation, 05(03), 1-2. https://doi.org/10.4172/21556199.1000e 146

Bingol, D., Tekin, N., \& Alkan, M. (2010). Brilliant Yellow dye adsorption onto sepiolite using a full factorial design. Applied Clay Science, 50(3), 315-321. https://doi.org/10.1016/j.clay.2010.08.015

Cortazar Martínez, A., Coronel Olivares, C., Escalante, A., \& González Ramírez, C. (2014). Contaminación generada por colorantes de la industria textil. Vida Científica, 2(3). https://doi.org/10.1080/07409710590915409

Crini, G. (2015). Non-conventional Adsorbents for Dye Removal, 359-407.

De Pablo, L. (1964). Las Arcillas. 1. Clasificación, identificación, usos y especificaciones industriales. Boletín de La Sociedad Geológica Mexicana, XXVII, 49-92. Retrieved from http://boletinsgm.igeolcu.unam.mx/bsgm/vols/epoca03/2702/de-pablo.pdf

Garzón, R. (2009). CINÉTICA DE DEGRADACIÓN DE COLORANTES TEXTILES DE DIFERENTES CLASES QUÍMICAS POR HONGOS Y BACTERIAS INMOVILIZADOS SOBRE FIBRA DE Agave tequilana Webber var. Azul. North. Instituto Politécnico Nacional.

Ghosh, D., \& Bhattacharyya, K. G. (2002). Adsorption of methylene blue on kaolinite. Applied Clay Science, 20(6), 295-300. https://doi.org/10.1016/S0169-1317(01)00081-3

Heller-Kallai, L. (2013a). Thermally modified clay minerals. In Developments in Clay Science (2nd ed., Vol. 5, pp. 411-433). Jerusalem: Elsevier Ltd. https://doi.org/10.1016/B978-008-098258-8.00014-6

Heller-Kallai, L. (2013b). Thermally modified clay minerals. Developments in Clay Science (2nd ed., Vol. 5). Jerusalem: Elsevier Ltd. https://doi.org/10.1016/B978-0-08-098258- 
8.00014-6

Hor, K. Y., Chee, J. M. C., Chong, M. N., Jin, B., Saint, C., Poh, P. E., \& Aryal, R. (2016). Evaluation of physicochemical methods in enhancing the adsorption performance of natural zeolite as low-cost adsorbent of methylene blue dye from wastewater. Journal of Cleaner Production, 118, 197-209. https://doi.org/10.1016/j.jclepro.2016.01.056

INEN. (2013). Agua, calidad de agua, muestreo manejo y conservación de muestras. 2169. Retrieved from http://sut.trabajo.gob.ec/publico/Normativa Técnica INEN/NTE INEN 2169 - AGUA. CALIDAD DEL AGUA. MUESTREO. MANEJO Y CONSERVACIÓN DE MUESTRAS.pdf

Instituto Ecuatoriano de Normalización. (2010). 695. Retrieved from http://normaspdf.inen.gob.ec/pdf/nte/695-1.pdf

Iyim, T. B., \& Güçlü, G. (2009). Removal of basic dyes from aqueous solutions using natural clay. Desalination, 249(3), 1377-1379. https://doi.org/10.1016/j.desal.2009.06.020

Kausar, A., Iqbal, M., Javed, A., Aftab, K., Nazli, Z.-H., Bhatti, H. N., \& Nouren, S. (2018a). Dyes adsorption using clay and modified clay: A review. Journal of Molecular Liquids, 256, 395-407. https://doi.org/10.1016/J.MOLLIQ.2018.02.034

Kausar, A., Iqbal, M., Javed, A., Aftab, K., Nazli, Z. i. H., Bhatti, H. N., \& Nouren, S. (2018b). Dyes adsorption using clay and modified clay: A review. Journal of Molecular Liquids, 256, 395-407. https://doi.org/10.1016/j.molliq.2018.02.034

Khan, T. A., Dahiya, S., \& Ali, I. (2012). Use of kaolinite as adsorbent: Equilibrium, dynamics and thermodynamic studies on the adsorption of Rhodamine B from aqueous solution. Applied Clay Science, 69, 58-66. https://doi.org/10.1016/j.clay.2012.09.001

Komadel, P., \& Madejová, J. (2013). Acid Activation of Clay Minerals. In Handbook of Clay Science (2nd ed., Vol. 5, pp. 385-409). Bratislava: Elsevier Ltd. https://doi.org/10.1016/B978-0-08-098258-8.00013-4

Larenas Herdoíza, D., Fierro-Renoy, V., \& Fierro-Renoy, C. (2017). Minería a Gran Escala: Una Nueva Industria para Ecuador. Polémika, 5(12), 67-91. Retrieved from https://www.usfq.edu.ec/publicaciones/polemika/Paginas/revistas/polemika012.aspx

Llive Carrillo, W. R. (2012). Análisis y evaluación de una planta de tratamiento de aguas residuales de una industria textil. Retrieved from http://repositorio.usfq.edu.ec/handle/23000/1968 
Martínez, Y. (2017). Evaluación sobre el uso de arcillas para la adsorción de colorantes utilizados en la industria textil (tesis doctoral). Universidad Nacional de la Plata. Universidad Nacional de la Plata.

Ministerio de Minería de Ecuador. (2016). Plan Nacional de Desarrollo del Sector Minero. Quito-Ecuador: Ministerio de Minería. Retrieved from https://drive.google.com/file/d/0B9t02UvtK83SbDA3a1FwZmpBY1k/view

Ministerio del Ambiente. (2015). Tulsma. Ecuador.

Moreno, J. C. (2007). Diseño de un sistema de purificacion de agua contaminada para su reutilización en usos industriales: Rama Textil. Universidad Dr. José Matías Delgado.

Ngulube, T., Gumbo, J. R., Masindi, V., \& Maity, A. (2017a). An update on synthetic dyes adsorption onto clay based minerals: A state-of-art review. Journal of Environmental Management, 191, 35-57. https://doi.org/10.1016/j.jenvman.2016.12.031

Ngulube, T., Gumbo, J. R., Masindi, V., \& Maity, A. (2017b). An update on synthetic dyes adsorption onto clay based minerals: A state-of-art review. Journal of Environmental Management, 191, 35-57. https://doi.org/10.1016/J.JENVMAN.2016.12.031

Re, H. (2010). Mejoramiento en la calidad de las aguas residuales de la planta de tratamiento de TEJIDOS PINTEX S.A. para su reutilización en los procesos industriales. Universidad de las Americas. Retrieved from http://dspace.udla.edu.ec/handle/33000/2287

Redacción Sierra Centro. (2010, July 14). 4 ríos de Chimborazo, contaminados |El Comercio. El Comercio. Retrieved from https://www.elcomercio.com/tendencias/rios-chimborazocontaminados.html

Saif Ur Rehman, M., Munir, M., Ashfaq, M., Rashid, N., Nazar, M. F., Danish, M., \& Han, J. I. (2013). Adsorption of Brilliant Green dye from aqueous solution onto red clay. Chemical Engineering Journal, 228, 54-62. https://doi.org/10.1016/j.cej.2013.04.094

Sanz, F. (2015). TRATAMIENTO DE AGUAS TEXTILES INDUSTRIALES MEDIANTE FOTOCATÁLISIS SOLAR Y REUTILIZACIÓN EN NUEVAS TINTURAS. Universidad Politécnica de Valencia.

Stawiński, W., Węgrzyn, A., Freitas, O., Chmielarz, L., Mordarski, G., \& Figueiredo, S. (2017). Simultaneous removal of dyes and metal cations using an acid, acid-base and base modified vermiculite as a sustainable and recyclable adsorbent. Science of the Total Environment, 576, 398-408. https://doi.org/10.1016/j.scitotenv.2016.10.120 
Toor, M., \& Jin, B. (2012). Adsorption characteristics, isotherm , kinetics , and diffusion of modified natural bentonite for removing diazo dye. Chemical Engineering Journal, 187, 79-88. https://doi.org/10.1016/j.cej.2012.01.089

Toor, M., Jin, B., Dai, S., \& Vimonses, V. (2015). Activating natural bentonite as a costeffective adsorbent for removal of Congo-red in wastewater. Journal of Industrial and Engineering Chemistry, 21, 653-661. https://doi.org/10.1016/j.jiec.2014.03.033

Turabik, M. (2008). Adsorption of basic dyes from single and binary component systems onto bentonite: Simultaneous analysis of Basic Red 46 and Basic Yellow 28 by first order derivative spectrophotometric analysis method. Journal of Hazardous Materials, 158(1), 52-64. https://doi.org/10.1016/j.jhazmat.2008.01.033

Uribe, R. A. (2015). Investigaciones de Materias Primas Minerales No Metálicas en el Ecuador. Revista Politécnica, 36(3), 34-44. Retrieved from http://www.revistapolitecnica.epn.edu.ec/images/revista/volumen36/tomo3/Investigacion esdeMateriasPrimasMinerales.pdf

Wang, W., Wang, F., Kang, Y., \& Wang, A. (2015). Enhanced Adsorptive Removal of Methylene Blue from Aqueous Solution by Alkali-Activated Palygorskite. Water, Air, \& Soil Pollution, 226(3), 83. https://doi.org/10.1007/s11270-015-2355-0

Yagub, M. T., Sen, T. K., Afroze, S., \& Ang, H. M. (2014). Dye and its removal from aqueous solution by adsorption: A review. Advances in Colloid and Interface Science, 209, 172184. https://doi.org/10.1016/J.CIS.2014.04.002

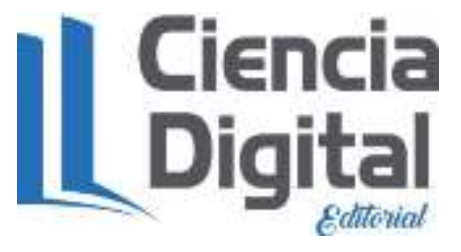




\section{PARA CITAR EL ARTÍCULO INDEXADO.}

Lara Hernández, A., Calderón, H., Ramos Flores., M., \& Ramos Flores, M. E. (2019). Evaluación de la capacidad de mineral aluminosilicato de la localidad de Cacha para la remoción de color en efluentes de la industria de lavado de blue jeans. Ciencia Digital, 3(3), 409-421. https://doi.org/10.33262/cienciadigital.v3i3.654

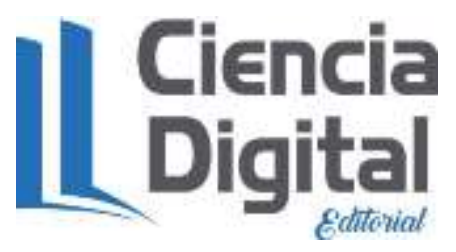

El artículo que se publica es de exclusiva responsabilidad de los autores y no necesariamente reflejan el pensamiento de la Revista Ciencia Digital.

El artículo queda en propiedad de la revista y, por tanto, su publicación parcial y/o total en otro medio tiene que ser autorizado por el director de la Revista Ciencia Digital.
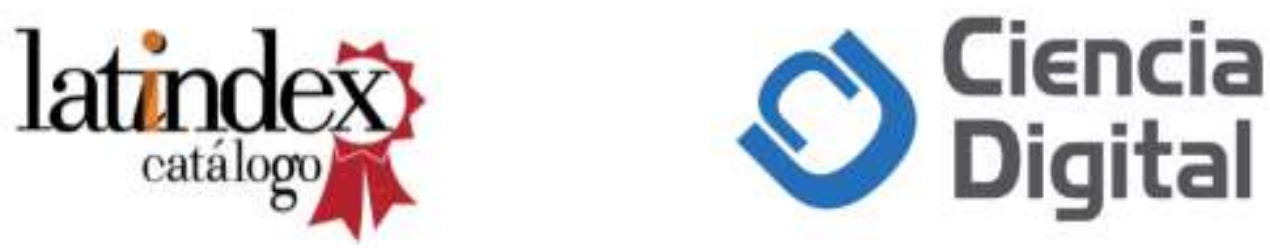